\title{
ORIENTED GORDIAN DISTANCE OF TWO-COMPONENT LINKS WITH UP TO SIX CROSSINGS
}

\section{SAORI KANENOBU AND TAIZO KANENOBU}

\begin{tabular}{|c|l|}
\hline Citation & OCAMI Preprint Series \\
\hline Issue Date & 2014 \\
\hline Type & Preprint \\
\hline Textversion & Author \\
\hline Rights & $\begin{array}{l}\text { C } 2015 \text { World Scientific Publishing Company. For personal use only. No other } \\
\text { uses without permission. }\end{array}$ \\
\hline Relation & $\begin{array}{l}\text { Preprint of an article submitted for consideration in Journal of Knot Theory and } \\
\text { Its Ramifications } @ 2015 \text { World Scientific Publishing Company. } \\
\text { https://www.worldscientific.com/worldscinet/jktr . }\end{array}$ \\
\hline Is version of & $\underline{\text { https://doi.org/10.1142/S0218216515400131 }}$ \\
\hline
\end{tabular}

From: Osaka City University Advanced Mathematical Institute http://www.sci.osaka-cu.ac.jp/OCAMI/publication/preprint/preprint.html 


\title{
ORIENTED GORDIAN DISTANCE OF TWO-COMPONENT LINKS WITH UP TO SIX CROSSINGS
}

\author{
SAORI KANENOBU AND TAIZO KANENOBU
}

Dedicated to the memory of Professor Fujitsugu Hosokawa

\begin{abstract}
The oriented Gordian distance between two oriented links is the minimal number of crossing changes needed to deform one into the other. We compile a table of oriented Gordian distances between 2-component non-splittable links with up to six crossings. In particular, we give a criterion of oriented Gordian distance two using a special value of the Jones polynomial, which allows us to prove that the unlinking number of the 2-component link $9_{3}^{2}$ is 3 . This is one of the 5 links for which Kohn could not compute the unlinking number.
\end{abstract}

\section{INTRODUCTION}

The oriented Gordian distance between two links $L$ and $M$ is the minimal number of crossing changes needed to deform $L$ into $M$, which we denote by $\overrightarrow{\mathrm{d}}(L, M)$. The main result in this note is Table 1, which lists the oriented Gordian distances of the nonsplittable 2-component links with up to 6 crossings; these are links as shown in Fig. 1 and their relatives; the mirror images and the links obtained by reversing the orientation of the component.



$H_{-}=2_{1}^{2}$

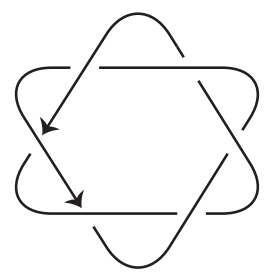

$T_{6}=6_{1}^{2}$

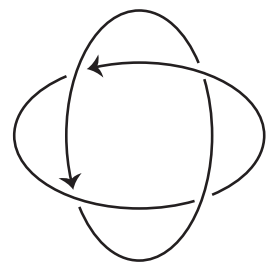

$T_{4}=4_{1}^{2}$

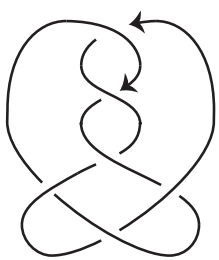

$6_{2}^{2}$

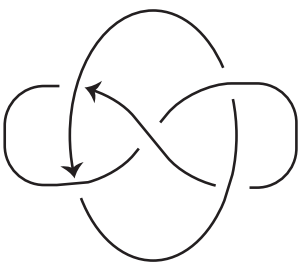

$5_{1}^{2}$

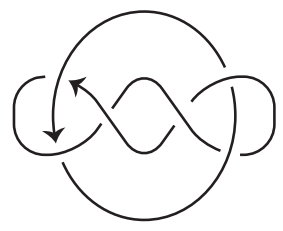

$6_{3}^{2}$



$3_{1} \# H_{-}$

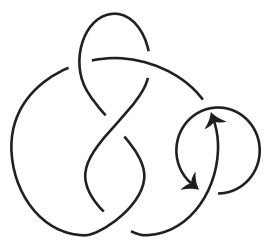

$4_{1} \# H_{-}$

FiguRE 1. 2-compoent oriented links with up to 6 crossings.

Date: December 7, 2014.

1991 Mathematics Subject Classification. Primary 57M25; Secondary 57M27. 
TABLE 1. Oriented Gordian distances of links with up to six crossings.

\begin{tabular}{|c|c|c|c|c|c|c|c|c|c|c|c|c|}
\hline & $H_{-}$ & $T_{4}$ & $T_{4}^{\prime}$ & & $3_{1} \# H_{-}$ & $3_{1} \# H_{+}$ & $T_{6}$ & $T_{6}^{\prime}$ & $6_{2}^{2}$ & $6_{3}^{2}$ & $6_{3}^{2 \prime}$ & $4_{1} \# H_{-}$ \\
\hline$U^{2}$ & 1 & 2 & 2 & 1 & 2 & 2 & 3 & 3 & 3 & 2 & 2 & 2 \\
\hline$H_{-}=2_{1}^{2}$ & 0 & 1 & 3 & 1 & 1 & 3 & 2 & 4 & 2 & 1 & 3 & 1 \\
\hline$H_{+}$ & 2 & 3 & 1 & 1 & 3 & 1 & 4 & 2 & 4 & 3 & 1 & 3 \\
\hline$T_{4}=4_{1}^{2}$ & & 0 & 4 & 2 & 2 & 4 & 1 & 5 & 1 & 1 & 4 & 2 \\
\hline$T_{4} !$ & & 4 & 1 & 2 & 4 & 2 & 5 & 2 & 5 & 4 & 2 & 4 \\
\hline$T_{4}^{\prime}$ & & & 0 & 2 & 4 & 2 & 5 & 1 & 5 & 4 & 1 & 4 \\
\hline$T_{4}^{\prime} !$ & & & & 2 & 2 & 4 & 2 & 5 & 1 & 2 & 4 & 2 \\
\hline $5_{1}^{2}=5_{1}^{2^{\prime}}$ & & & & 0 & 2 & 2 & 3 & 3 & 3 & 2 & 2 & 2 \\
\hline $5_{1}^{2} !$ & & & & 2 & 2 & 2 & 3 & 3 & 3 & 2 & 2 & 2 \\
\hline $3_{1} \# H_{-}$ & & & & & 0 & 2 & 3 & 5 & 3 & 2 & 4 & 2 \\
\hline $3_{1}$ !\# $H_{+}$ & & & & & 4 & 2 & 5 & 3 & 5 & 4 & 2 & 4 \\
\hline $3_{1} \# H_{+}$ & & & & & & 0 & 5 & 3 & 5 & 4 & 2 & 4 \\
\hline $3_{1} ! \# H_{-}$ & & & & & & & 3 & 5 & 3 & 2 & 4 & 2 \\
\hline$T_{6}=6_{1}^{2}$ & & & & & & & 0 & 6 & 1 & 1 & 5 & 3 \\
\hline$T_{6} !$ & & & & & & & 6 & 2 & 6 & 5 & 3 & 5 \\
\hline$T_{6}^{\prime}$ & & & & & & & & 0 & 6 & 5 & 1 & 5 \\
\hline$T_{6}^{\prime} !$ & & & & & & & & & 1 & 3 & 5 & 3 \\
\hline $6_{2}^{2}$ & & & & & & & & & 0 & 2 & 5 & 3 \\
\hline $6_{2}^{2} !=6_{2}^{2 \prime}$ & & & & & & & & & 6 & 5 & 2 & 5 \\
\hline $6_{3}^{2}$ & & & & & & & & & & 0 & 4 & 2 \\
\hline $6_{3}^{2} !$ & & & & & & & & & & 4 & 2 & 4 \\
\hline $6_{3}^{2^{\prime}}$ & & & & & & & & & & & 0 & 4 \\
\hline $6_{3}^{2 \prime} !$ & & & & & & & & & & & & 2 \\
\hline $4_{1} \# H_{+}$ & & & & & & & & & & & & 2 \\
\hline
\end{tabular}

TABLE 2. Unoriented Gordian distances of links with up to six crossings.

\begin{tabular}{l|cccccccc}
\hline & $H$ & $T_{4}$ & $5_{1}^{2}$ & $3_{1} \# H$ & $T_{6}$ & $6_{2}^{2}$ & $6_{3}^{2}$ & $4_{1} \# H$ \\
\hline$U^{2}$ & 1 & 2 & 1 & 2 & 3 & 3 & 2 & 2 \\
$H=2_{1}^{2}$ & 0 & 1 & 1 & 1 & 2 & 2 & 1 & 1 \\
$T_{4}=4_{1}^{2}$ & & 0 & 2 & 2 & 1 & 1 & 1 & 2 \\
$T_{4} !$ & & 1 & 2 & 2 & 2 & 1 & 2 & 2 \\
$5_{1}^{2}$ & & & 2 & 3 & 3 & 2 & 2 \\
$5_{1}^{2} !$ & & & 2 & 3 & 3 & 2 & 2 \\
$3_{1} \# H$ & & & 0 & 3 & 3 & 2 & 2 \\
$3_{1} ! \# H$ & & & 2 & 3 & 3 & 2 & 2 \\
$T_{6}=6_{1}^{2}$ & & & & 0 & 1 & 1 & 3 \\
$T_{6} !$ & & & & 2 & 1 & 3 & 3 \\
$6_{2}^{2}$ & & & & & 0 & 2 & 3 \\
$6_{3}^{2}$ & & & & & & & 0 & 2 \\
$6_{3}^{2} !$ & & & & & & & 2 & 2 \\
\hline
\end{tabular}

We denote by $U^{c}$ the trivial $c$-component link; in particular, we denote the trivial knot by $U\left(=U^{1}\right)$. For a link $L$, we denote by $L$ ! its mirror image. For oriented links $L$ and $M$ we denote their split union and product by $L \sqcup M$ and $L \# M$, respectively. For knots and links with up to 9 crossings we use Rolfsen notations [12]; more precisely, $c_{n}^{2}$ denotes oriented 2-component links of non-positive linking number with diagram as in 
Appendix $\mathrm{C}$ of [12], and $c_{n}^{2 \prime}$ denotes the oriented links obtained from $c_{n}^{2}$ by reversing the orientation of one component. Specifically, we denote the positive and negative Hopf links by $H_{+}$and $H_{-}$, respectively, and so $H_{+}=2_{1}^{2} !=2_{1}^{2 \prime}$ and $H_{-}=2_{1}^{2}=2_{1}^{2} !^{\prime}$. Also, $T_{2 n}$ denotes the torus link of type $(2,2 n)$ with linking number $-n$ and parallel orientation, and $T_{2 n}^{\prime}$ and $T_{2 n}^{\prime}$ ! denote the torus links of type $(2,2 n)$ obtained from $T_{2 n}$ and $T_{2 n}$ !, respectively, by changing the orientation of one component, and so $T_{4}=4_{1}^{2}$ and $T_{6}=6_{1}^{2}$; cf. $[4$, Sec. 5.1], [5, Table 2], [6, Table 6].

Notice that for links $L$ and $M$ in Table 1 the following hold: $\overrightarrow{\mathrm{d}}(L, M)=\overrightarrow{\mathrm{d}}(L !, M !)=$ $\overrightarrow{\mathrm{d}}\left(L^{\prime}, M^{\prime}\right)=\overrightarrow{\mathrm{d}}\left(L^{\prime} !, M^{\prime} !\right)$.

Similarly, for unoriented links we define the unoriented Gordian distance; we denote by $\mathrm{d}(L, M)$ the Gordian distance of unoriented links $L$ and $M$. For links $L$ and $M$ in Table 1 we have $\mathrm{d}(L, M)=\min \left\{\overrightarrow{\mathrm{d}}(L, M), \overrightarrow{\mathrm{d}}\left(L^{\prime}, M\right)\right\}$, which yields Table 2 compiling the unoriented Gordian distances of non-splittable 2-component links with up to 6 crossings, where $H$ denotes the unoriented Hopf link.

For many pairs of 2-component links with up to 6 crossings we can decide the Gordian distances by the linking numbers and Gordian distances of components (Proposition 2.1). Other cases are decided by the signature (Propositions 2.3 and 2.4), the special value of the Jones polynomial (Proposition 3.1 and Theorem 3.4), and the criterion for 2-bridge links with unoriented Gordian distance one (Proposition 4.1) due to Torisu [15] and Darcy and Sumners [1].

Traczyk [16] has proved that the unknotting number of the knot $7_{4}$ is two using the special value of the Jones polynomial as follows: Suppose that the knot $7_{4}$ is unknotted by a single crossing change. Then since the signature of $7_{4}$ is 2 , that crossing should be negative. However, since the value of the Jones polynomial of $7_{4}$ at $t=e^{i \pi / 3}$ is $i \sqrt{3}$, that crossing should be positive, a contradiction. Developing such a discussion, we have several criteria for the unknotting number and Gordian distance for knots and links as well as the band-unknotting number and band-Gordian distance using the value of the Jones polynomial at $t=e^{i \pi / 3}$; see $[4,5,6,8,14]$ and also compare [9]. In particular, Theorem 3.4 is a criterion of oriented Gordian distance two, which allows us to prove that the unlinking number of the 2-component link $9_{3}^{2}$ is 3 (Fig. 4). This link is one of the five 2-component prime links with up to 9 crossings whose unlinking number had not been settled in the table of unlinking numbers compiled by Kohn [7].

This note is organized as follows: In Sect. 2 we give an elementary lower bound of the Gordian distance and some properties of the signature. In Sect. 3 we give some methods to give a lower bound of the Gordian distance using the Jones polynomial. In Sect. 4 we review Torisu, Darcy and Sumners' criterion for 2-bridge links with unoriented Gordian distance one. In Sect. 5 we explain the method for compiling Table 1, the table of the oriented Gordian distances of 2-component links with up to 6 crossings.

\section{Oriented Gordian distance}

Given two oriented links $L$ and $M$ with the same number of components, we define the oriented Gordian distance of $L$ and $M, \vec{d}(L, M)$, to be the minimal number of crossing changes needed to deform $L$ into $M$. Similarly, for unoriented links we define the (unoriented) Gordian distance, which we denote by $\mathrm{d}(L, M)$, where the orientations 
of $L$ and $M$ are ignored, and thus, $\mathrm{d}(L, M) \leq \overrightarrow{\mathrm{d}}(L, M)$. In particular, the unlinking number of $L$ (or the unknotting number if $L$ is a knot), $\mathrm{u}(L)$, is the Gordian distance from $L$ to the trivial link; $\mathrm{u}(L)=\mathrm{d}\left(L, U^{c}\right)=\overrightarrow{\mathrm{d}}\left(L, U^{c}\right)$, where $U^{c}$ is the $c$-component trivial link with $c$ the number of the components of $L$.

For three oriented links $L, M, N$, the triangle inequality holds:

$$
\overrightarrow{\mathrm{d}}(L, M) \leq \overrightarrow{\mathrm{d}}(L, N)+\overrightarrow{\mathrm{d}}(N, M) .
$$

In particular, we have:

$$
\overrightarrow{\mathrm{d}}(L, M) \leq \mathrm{u}(L)+\mathrm{u}(M) .
$$

The following is a generalization of Eq. (1) in [7].

Proposition 2.1. Let $L=L_{1} \cup L_{2}$ and $M=M_{1} \cup M_{2}$ be oriented 2-component links. Then

(3) $\overrightarrow{\mathrm{d}}(L, M) \geq \min \left\{\overrightarrow{\mathrm{d}}\left(L_{1}, M_{1}\right)+\overrightarrow{\mathrm{d}}\left(L_{2}, M_{2}\right), \overrightarrow{\mathrm{d}}\left(L_{1}, M_{2}\right)+\overrightarrow{\mathrm{d}}\left(L_{2}, M_{1}\right)\right\}+|\operatorname{lk}(L)-\operatorname{lk}(M)|$, where $\operatorname{lk}(L)$ is the linking number of $L ; \operatorname{lk}(L)=\operatorname{lk}\left(L_{1}, L_{2}\right)$.

If $K_{1}$ or $K_{2}$ is an invertible knot, then $\overrightarrow{\mathrm{d}}\left(K_{1}, K_{2}\right)=\mathrm{d}\left(K_{1}, K_{2}\right)$. In this paper, we consider knots and links with small crossing numbers, and so we treat only an invertible knot.

Two oriented links $L$ and $M$ are related by a coherent band surgery if there exists an embedding $b: I \times I \rightarrow S^{3}$ such that $L \cap b(I \times I)=b(I \times \partial I)$ and $M \cap b(I \times I)=b(\partial I \times I)$, and that $L \backslash b(I \times \partial I)$ and $M \backslash b(\partial I \times I)$ have the compatible orientation, where $I$ is the unit interval $[0,1]$. The following are basic properties of the link signature (Lemmas 7.1, 7.2 and Corollary 7.4 in [10]).

Proposition 2.2. (i) If two links $L$ and $M$ are related by a coherent band surgery, then

$$
|\sigma(L)-\sigma(M)| \leq 1 .
$$

(ii) For oriented links $L$ and $M$, we have

$$
\begin{aligned}
\sigma(L \sqcup M) & =\sigma(L)+\sigma(M) ; \\
\sigma(L \# M) & =\sigma(L)+\sigma(M) .
\end{aligned}
$$

A skein triple is an ordered set of three oriented links that are identical except near one point where they are as in Fig. 2 , which we denote by $\left(L_{+}, L_{-}, L_{0}\right)$. Then we have the following; cf. [13, Eqs. (7) and (8)].

Proposition 2.3. Let $\left(L_{+}, L_{-}, L_{0}\right)$ be a skein triple. Then

$$
\begin{aligned}
\sigma\left(L_{ \pm}\right)-\sigma\left(L_{0}\right) & \in\{-1,0,1\} \\
\sigma\left(L_{-}\right)-\sigma\left(L_{+}\right) & \in\{0,1,2\} .
\end{aligned}
$$

Proof. Equation (7) is just Proposition 2.2(i). Since the link $L_{-}$is obtained from $L_{+}$by changing a positive crossing, $L_{+}$yields the product link $L_{-} \# H_{+}$by doing a coherent band surgery as shown in Fig. 3, where $H_{+}$is the positive Hopf link. Then by Eq. (4) we have

$$
\left|\sigma\left(L_{+}\right)-\sigma\left(L_{-} \# H_{+}\right)\right| \leq 1
$$


Since

$$
\sigma\left(L_{-} \# H_{+}\right)=\sigma\left(L_{-}\right)+\sigma\left(H_{+}\right)=\sigma\left(L_{-}\right)-1
$$

by Eq. (6), we obtain Eq. (8).

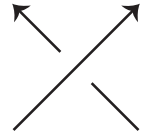

$L_{+}$

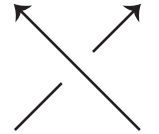

$L_{-}$

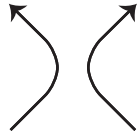

$L_{0}$

FiguRE 2. Skein triple.

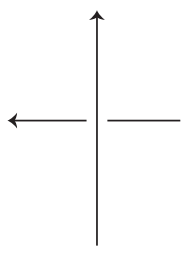

$L_{+}$
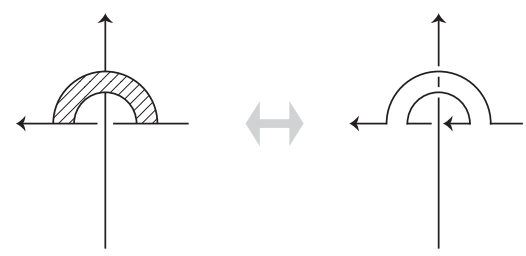

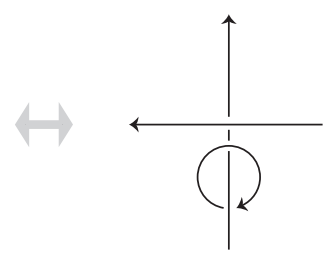

$L_{-} \# H_{+}$

Figure 3. Two links $L_{+}$and $L_{-} \# H_{+}$are related by a coherent band surgery.

The following is immediate from Eq. (8) in Proposition 2.3; cf. Theorem 10.1 in [10].

Proposition 2.4. For two oriented links $L, M$ with the same number of components, we have

$$
\overrightarrow{\mathrm{d}}(L, M) \geq|\sigma(L)-\sigma(M)| / 2 .
$$

Example 2.5. $\overrightarrow{\mathrm{d}}\left(T_{4} !, T_{6}^{\prime}\right)>1$. Let $L=T_{4}$ ! and $M=T_{6}^{\prime}$. Suppose that $\overrightarrow{\mathrm{d}}(L, M)=1$. Since $\operatorname{lk}(L)=2, \operatorname{lk}(M)=3, L$ is obtained from $M$ by changing a positive crossing. However, since $\sigma(L)=-3$ and $\sigma(M)=-1$, by Eq. (8) in Prosposition $2.3 L$ is obtained from $M$ by changing a negative crossing, a contradiction. So, only using either Proposition 2.1 or Proposition 2.4, we cannot obtain the result.

On the other hand, $\overrightarrow{\mathrm{d}}\left(L^{\prime}, M^{\prime}\right)>1$ is obtained only from Proposition 2.4. In fact, $\sigma\left(L^{\prime}\right)=1, \sigma\left(M^{\prime}\right)=5$. Notice that for a link $L$, the value $\sigma(L)+\operatorname{lk}(L)$ is an invariant of an unoriented link type (Theorem 1 of [11]), which implies that $\sigma\left(L^{\prime}\right)=\sigma(L)+2 \operatorname{lk}(L)$.

$\overrightarrow{\mathrm{d}}\left(6_{3}^{2} !, T_{6}^{\prime}\right)=\overrightarrow{\mathrm{d}}\left(6_{3}^{2 \prime} !, T_{6}\right)>1$ is similarly obtained. In fact, $\sigma\left(6_{3}^{2}\right)=\sigma\left(T_{4}\right)=3$ and $\operatorname{lk}\left(6_{3}^{2}\right)=\operatorname{lk}\left(T_{4}\right)=-2$. 


\section{JONES POLYNOMIAL}

The Jones polynomial $V(L ; t) \in \boldsymbol{Z}\left[t^{ \pm 1 / 2}\right][2]$ of an oriented link $L$ is defined by the following formulas:

$$
\begin{gathered}
V(U ; t)=1 \\
t^{-1} V\left(L_{+} ; t\right)-t V\left(L_{-} ; t\right)=\left(t^{1 / 2}-t^{-1 / 2}\right) V\left(L_{0} ; t\right) ;
\end{gathered}
$$

where $U$ is the unknot and $\left(L_{+}, L_{-}, L_{0}\right)$ is a skein triple.

Jones [3] has shown:

$$
V(L ; \omega)= \pm i^{c-1}(i \sqrt{3})^{\delta},
$$

where $\omega=e^{i \pi / 3}, V(L ; \omega)$ means the value of $V(L ; t)$ at $t^{1 / 2}=e^{i \pi / 6}, c$ is the number of the components of $L$, and $\delta=\operatorname{dim} H_{1}\left(\Sigma_{2}(L) ; \boldsymbol{Z}_{3}\right)$ with $\Sigma_{2}(L)$ the 2-fold covering space of $S^{3}$ branched over $L$.

The following criterion is useful to decide the oriented Gordian distance; (i) is Proposition 4.2 in [8]; cf. [6, 14, 16], and (ii) is Theorem 5.2(ii) in [6].

Proposition 3.1. (i) If a link $M$ is obtained from a link $L$ by changing a positive crossing of $L$, then

$$
V(L ; \omega) / V(M ; \omega) \in\left\{ \pm 1, i \sqrt{3}^{ \pm 1}\right\} .
$$

(ii) Suppose that two c-component links $L$ and $M$ are related by a crossing change. If $V(L ; \omega)=\eta V(M ; \omega)= \pm i^{c-1}(i \sqrt{3})^{\delta}, \eta= \pm 1$, then $i^{c-1} V(L ;-1) \equiv i^{c-1} \eta V(M ;-1)$ $\left(\bmod 3^{\delta+1}\right)$.

Example 3.2. (i) $\overrightarrow{\mathrm{d}}\left(6_{2}^{2}, 6_{3}^{2}\right)>1$. Let $L=6_{2}^{2}$ and $M=6_{3}^{2}$. Suppose that $\overrightarrow{\mathrm{d}}(L, M)=1$. Then since $\operatorname{lk}(L)=-3$ and $\operatorname{lk}(M)=-2, L$ is obtained from $M$ by changing a positive crossing. Then $V(M ; \omega) / V(L ; \omega)=-i \sqrt{3}$ contradicts Proposition 3.1(i).

(ii) $\overrightarrow{\mathrm{d}}\left(T_{4}^{\prime}, 6_{3}^{2}\right.$ !) $>1$. Let $L=T_{4}^{\prime}$ and $M=6_{3}^{2}$ !. Suppose that $\overrightarrow{\mathrm{d}}(L, M)=1$. Then since $\sigma(L)=-1$ and $\sigma(M)=-3, L$ is obtained from $M$ by changing a positive crossing. Then $V(M ; \omega) / V(L ; \omega)=-i \sqrt{3}$ contradicts Proposition 3.1(i).

(iii) $\overrightarrow{\mathrm{d}}\left(6_{3}^{2} !, 6_{3}^{2^{\prime}}\right)>1$. Let $L=6_{3}^{2}$ ! and $M=6_{3}^{2^{\prime}}$. Suppose that $\overrightarrow{\mathrm{d}}(L, M)=1$. Since $V(L ; \omega)=V(M ; \omega)=\sqrt{3}$, we have $i V(L ;-1) \equiv i V(M ;-1)\left(\bmod 3^{2}\right)$ by Proposition 3.1(ii). However, $i V(L ;-1)=-12$ and $i V(M ;-1)=12$, a contradiction.

Remark 3.3. (i) We can prove $\mathrm{d}\left(6_{2}^{2}, 6_{3}^{2}\right)>1$ by applying Proposition 7.1(i) in [6], a criterion using a special value of the Q polynomial; this method is due to Stoimenow [13, Theorem 4.1].

(ii) We can prove $\overrightarrow{\mathrm{d}}\left(T_{4}^{\prime}, 6_{3}^{2}\right.$ !) > 1 by applying Proposition 6.4(ii)(a) in [6], a criterion using a special value of the HOMFLYPT polynomial. However, this cannot prove $\overrightarrow{\mathrm{d}}\left(T_{4}, 6_{3}^{2 \prime} !\right)>1$.

Theorem 3.4. Let $L$ and $M$ be oriented c-component links with $\overrightarrow{\mathrm{d}}(L, M)=2$ and $\sigma(L)-\sigma(M)>2$. If $V(L ; \omega)=V(M ; \omega)= \pm i^{c-1}(i \sqrt{3})^{\delta}$, we have $i^{c-1} V(L ;-1) \equiv$ $i^{c-1} V(M ;-1)\left(\bmod 3^{\delta+1}\right)$. 
Proof. Let $N$ be an oriented link obtained from both $L$ and $M$ by a crossing change. Since $\sigma(L)-\sigma(M)>2$, we have $\sigma(L)-\sigma(N), \sigma(N)-\sigma(M)>0$, and so by Proposition 2.3 $N$ is obtained from $L$ by changing a negative crossing and also $N$ is obtained from $M$ by changing a positive crossing. Let $v_{0}=V(L ; \omega)=V(M ; \omega)= \pm i^{c-1}(i \sqrt{3})^{\delta}$. Then by Proposition 3.1(i) we have:

$$
V(N ; \omega) \in\left\{ \pm v_{0}, i \sqrt{3}^{ \pm 1} v_{0}\right\} \cap\left\{ \pm v_{0},-i \sqrt{3}^{ \pm 1} v_{0}\right\}=\left\{ \pm v_{0}\right\} .
$$

Thus put $V(N ; \omega)=\eta v_{0}, \eta= \pm 1$. Applying Proposition 3.1(ii), we have $\eta i^{c-1} V(N ;-1) \equiv$ $i^{c-1} V(L ;-1) \equiv i^{c-1} V(M ;-1)\left(\bmod 3^{\delta+1}\right)$, completing the proof.

Example 3.5. $\overrightarrow{\mathrm{d}}\left(T_{6}, 6_{3}^{2} !\right)>2$. Let $L=T_{6}$ and $M=6_{3}^{2}$ !. In Example 2.5, we have seen $\overrightarrow{\mathrm{d}}(L, M)>1$. Suppose that $\overrightarrow{\mathrm{d}}(L, M)=2$. We have $\sigma(L)=5, \sigma(M)=1, V(L ; \omega)=$ $V(M ; \omega)=\sqrt{3}, i V(L ;-1)=-6, i V(M ;-1)=-12$, contradicting Theorem 3.4.

In Table 3 we give some pairs of links to which we can apply Theorem 3.4. The pairs $\left(3_{1}, 7_{4}\right)$ and $\left(3_{1}, 3_{1}\right.$ ! \#4 1$)$ are only examples of knots with up to 7 crossings. For 2-component prime links with up to 9 crossings there remained five links whose unlinking number had not been settled in the table of Kohn [7] and using Theorem 3.4 we may prove $\mathrm{u}\left(9_{3}^{2}\right)=3$; see Fig. 4 , where by changing the 3 crossings near the asterisk "*" in the diagram we obtain the trivial link.

TABle 3. Pairs of links $L$ and $M$ with $\overrightarrow{\mathrm{d}}(L, M)=3$.

\begin{tabular}{rcrrrrr}
\hline$L$ & $M$ & $\sigma(L)$ & $\sigma(M)$ & $V(L ; \omega)=V(M ; \omega)$ & $V(L ;-1)$ & $V(M ;-1)$ \\
\hline $3_{1}$ & $7_{4}$ & 2 & -2 & $-i \sqrt{3}$ & -3 & -15 \\
$3_{1}$ & $3_{1} ! \# 4_{1}$ & 2 & -2 & $-i \sqrt{3}$ & -3 & -15 \\
$9_{3}^{2}$ & $U^{2}$ & 3 & 0 & $\sqrt{3}$ & $30 i$ & 0 \\
\hline
\end{tabular}

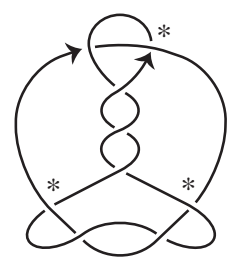

Figure 4. The link $9_{3}^{2}$.

\section{TORISU-DARCY-SUMNERS' CRITERION}

For relatively prime integers $p, q$, we let $S(p, q)$ denote any 2-bridge knot or link such that the 2-fold covering space of $S^{3}$ branched over $S(p, q)$ is homeomorphic to the lens space of type $(p, q)$. Notice that $S(p,-q)(=S(-p, q))$ is the mirror image of $S(p, q)$. Two unoriented 2-bridge link $S(p, q)$ and $S(r, s), p, r>0$, are isotopic if and only if $p=r$ and $q \equiv s^{ \pm 1}(\bmod p)$.

The following was independently proved by Darcy and Sumners [1] and Torisu [15]. 
Proposition 4.1. Two unoriented 2-bridge knots or links $S(p, q)$ and $S(r, s)$ are related by a crossing change if and only if: there exist pairs of relatively prime integers $(m, n)$ and $(a, b)$ such that $n \neq 0 ; r m+a n \neq 0 ; r b-s a=1$ and $S(p ; q)$ is isotopic to $S\left(2 a n^{2}+\right.$ $\left.r(2 m n \pm 1), 2 b n^{2}+s(2 m n \pm 1)\right)$.

Example 4.2. $\mathrm{d}\left(5_{1}^{2}, 5_{1}^{2} !\right)>1$. Suppose that $\mathrm{d}\left(5_{1}^{2}, 5_{1}^{2} !\right)=1$. Then since $5_{1}^{2}=S(8,3)$ and $5_{1}^{2} !=S(8,5)$, there exist pairs of prime integers $(m, n)$ and $(a, b)$ such that $n \neq 0$; $8 m+a n \neq 0 ; 8 b-5 a=1$ and $S(8,3)$ is isotopic to $S\left(2 a n^{2}+8(2 m n \pm 1), 2 b n^{2}+5(2 m n \pm 1)\right)$. Then $\left|2 a n^{2}+8(2 m n \pm 1)\right|=8$. Since $8 m+a n \neq 0$, we have $8 m n+a n^{2}= \pm 8$. Then $n$ is a divisor of 8 . However, since $a$ is odd, this cannot occur.

\section{Decision of the oriented Gordian distances}

Table 1 lists the oriented Gordian distances between two links with up to six crossings, which we compile according to the following procedures.

Step 1. Combining Eqs. (3) and (2), we have the inequalities:

$$
\begin{array}{r}
\max \left\{1, \min \left\{\overrightarrow{\mathrm{d}}\left(L_{1}, M_{1}\right)+\overrightarrow{\mathrm{d}}\left(L_{2}, M_{2}\right), \overrightarrow{\mathrm{d}}\left(L_{1}, M_{2}\right)+\overrightarrow{\mathrm{d}}\left(L_{2}, M_{1}\right)\right\}+|\operatorname{lk}(L)-\mathrm{lk}(M)|\right\} \\
\leq \overrightarrow{\mathrm{d}}(L, M) \leq \mathrm{u}(L)+\mathrm{u}(M)
\end{array}
$$

where $L=L_{1} \cup L_{2}$ and $M=M_{1} \cup M_{2}$ are inequivalent oriented 2-component links. We have the table of unlinking numbers for prime links with up to 9 crossings in [7], and $\mathrm{u}\left(3_{1} \# H_{-}\right)=\mathrm{u}\left(4_{1} \# H_{-}\right)=2$ are easy. Further, using the signature (Eq. (11)) we obtain sharper lower bounds:

- $\overrightarrow{\mathrm{d}}\left(T_{4}{ }^{\prime} !, T_{6}\right)=\overrightarrow{\mathrm{d}}\left(T_{4}{ }^{\prime}, T_{6} !\right)>1$, which implies $\overrightarrow{\mathrm{d}}\left(T_{4} !, T_{6}{ }^{\prime}\right)=\overrightarrow{\mathrm{d}}\left(T_{4}, T_{6}{ }^{\prime} !\right)>1$ (Example 2.5).

- $\overrightarrow{\mathrm{d}}\left(6_{3}^{2 \prime} !, T_{6}\right)=\overrightarrow{\mathrm{d}}\left(6_{3}^{2 \prime}, T_{6} !\right)>1$, which implies $\overrightarrow{\mathrm{d}}\left(6_{3}^{2} !, T_{6}{ }^{\prime}\right)=\overrightarrow{\mathrm{d}}\left(6_{3}^{2}, T_{6}{ }^{\prime} !\right)>1$ (Example 2.5$)$.

- $\overrightarrow{\mathrm{d}}\left(T_{6}, T_{6}{ }^{\prime} !\right)=\overrightarrow{\mathrm{d}}\left(T_{6}{ }^{\prime}, T_{6} !\right)>1$.

We have the table of signatures of links in [5, Table 2]. This yields Table 4, which lists $\overrightarrow{\mathrm{d}}(L, M)$ for the link $L$ in the column and $M$ in the row, and $p-q$ means that $p \leq \overrightarrow{\mathrm{d}}(L, M) \leq q$.

Step 2. We examine the pairs $(L, M)$ with $1 \leq \overrightarrow{\mathrm{d}}(L, M) \leq q, q>1$, in Table 4 . Then we obtain Table 5, where:

1) Changing a crossing in a minimal diagram of $L$ (resp. $M$ ), we obtain $M$ (resp. $L)$.

2) See Fig. 5 , where $L \rightarrow M$ means that the link $M$ is obtained from $L$ by changing the crossing near the asterisk "*" in the diagram of $L$ shown there.

3) See Example 4.2.

4) See Example 3.2.

5) See Example 3.5. 


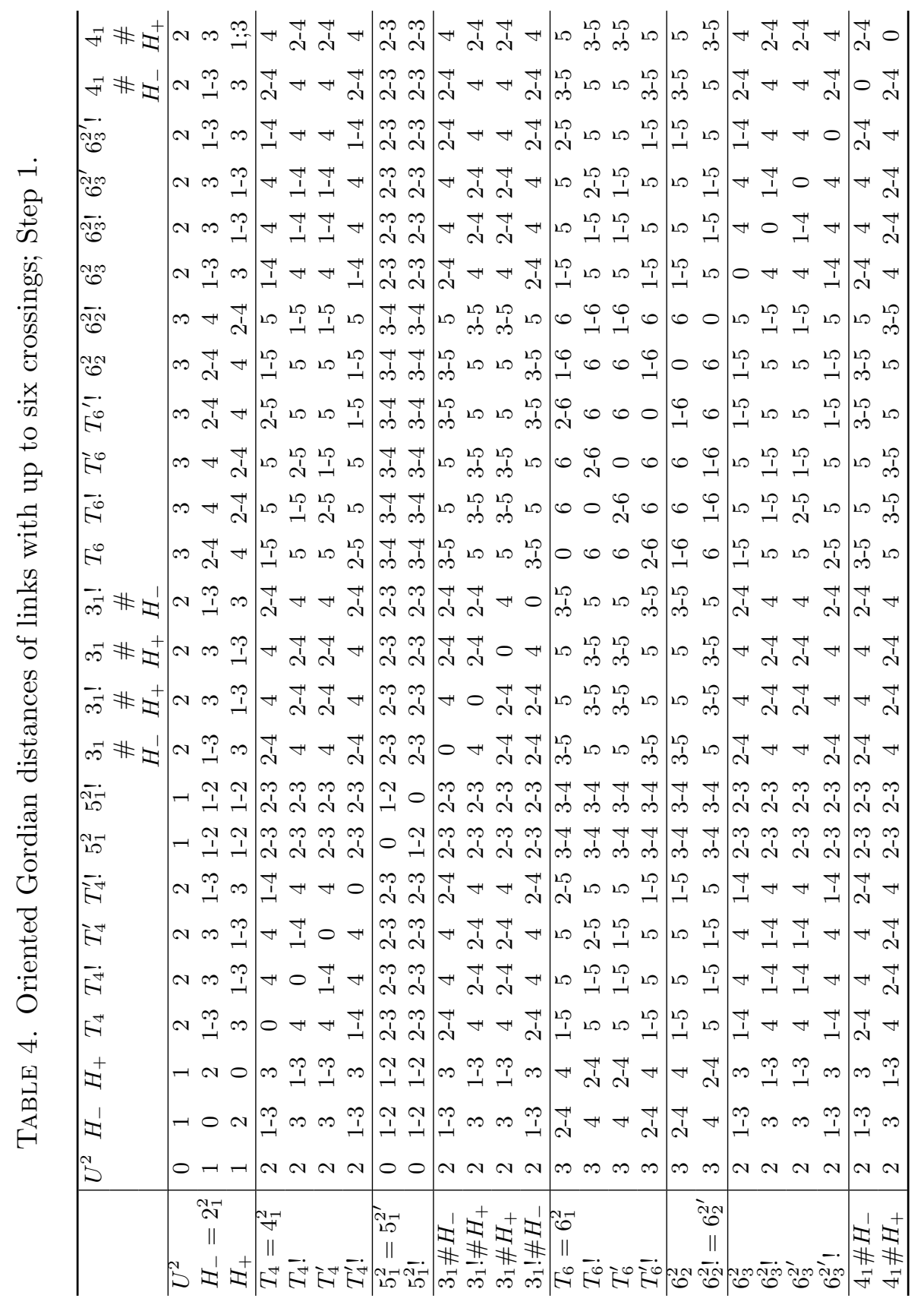




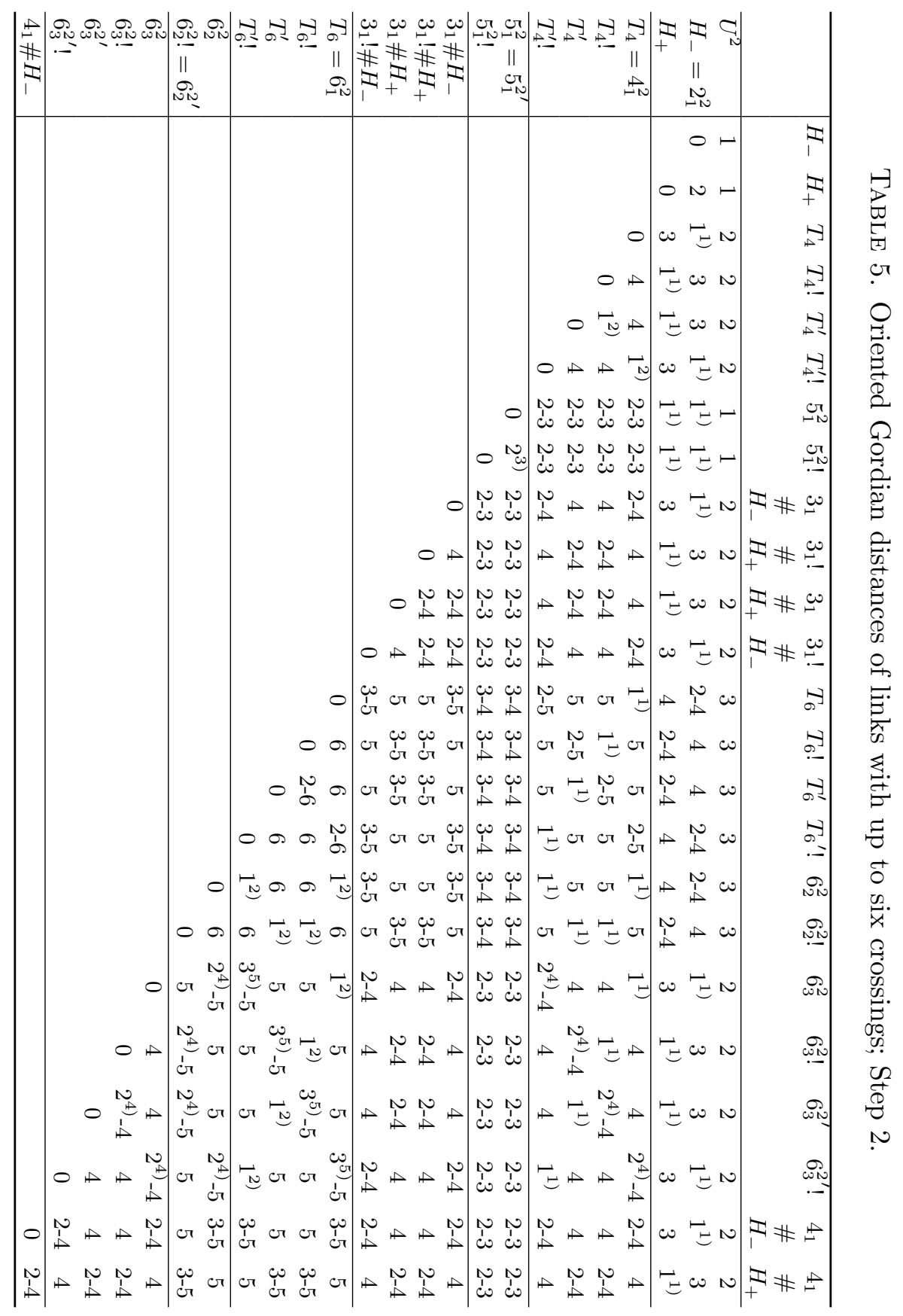




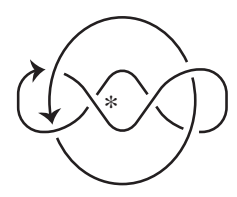

(a)

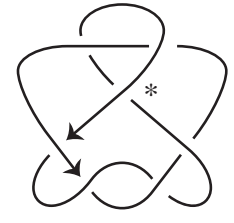

(b)

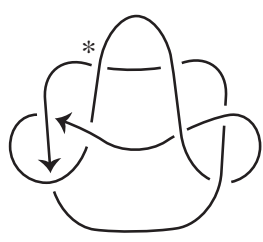

(c)

Figure 5. (a) $T_{4}^{\prime} \rightarrow T_{4}$ !, (b) $T_{6} \rightarrow 6_{2}^{2}$, (c) $6_{3}^{2} \rightarrow T_{6}$.

Step 3. We examine the pairs $(L, M)$ with $2 \leq \overrightarrow{\mathrm{d}}(L, M) \leq q, q>2$, in Table 5. Then we see they all are of oriented Gordian distance two since $\overrightarrow{\mathrm{d}}(L, N)=\overrightarrow{\mathrm{d}}(N, M)=1$ for some link $N$. This yields Table 6 , where:

6) $N=H_{-}$.

7) $N=H_{+}$.

8) $N=3_{1} \sqcup U$.

9) $N=3_{1} ! \sqcup U$.

10) $N=T_{4}$.

11) $N=T_{4} !$

12) $N=T_{4}^{\prime}$.

13) $N=T_{4}^{\prime}$ !.

14) $N=4_{1} \sqcup U$.

15) $N=6_{2}^{2}$.

16) $N=62$ !

Step 4. We examine the pairs $(L, M)$ with $3 \leq \overrightarrow{\mathrm{d}}(L, M) \leq q, q>3$, in Table 6 . Then we see they all are of oriented Gordian distance three since $\overrightarrow{\mathrm{d}}(L, M) \leq \overrightarrow{\mathrm{d}}\left(L, H_{ \pm}\right)+$ $\overrightarrow{\mathrm{d}}\left(H_{ \pm}, M\right)=3$. This yields Table 1 .

\section{ACKNOWLEDGEMENTS}

The second author was partially supported by KAKENHI, Grant-in-Aid for Scientific Research (C) (No. 21540092), Japan Society for the Promotion of Science.

\section{REFERENCES}

[1] Isabel K. Darcy and De Witt Sumners, Rational tangle distances on knots and links, Math. Proc. Cambridge Philos. Soc. 128 (2000), no. 3, 497-510.

[2] Vaughan F. R. Jones, Hecke algebra representations of braid groups and link polynomials, Ann. of Math. (2) 126 (1987), no. 2, 335-388.

[3] Vaughan F. R. Jones, On a certain value of the Kauffman polynomial, Comm. Math. Phys. 125 (1989), no. 3, 459-467.

[4] Taizo Kanenobu, Band surgery on knots and links, J. Knot Theory Ramifications 19 (2010), no. 12, $1535-1547$.

[5] Taizo Kanenobu, Band surgery on knots and links, II, J. Knot Theory Ramifications 21 (2012), no. 9, 1250086, 22.

[6] Taizo Kanenobu and Hiromasa Moriuchi, Links which are related by a band surgery or crossing change, Bol. Soc. Mat. Mex. (3) 20 (2014), no. 2, 467-483.

[7] Peter Kohn, Unlinking two component links, Osaka J. Math. 30 (1993), no. 4, 741-752. 


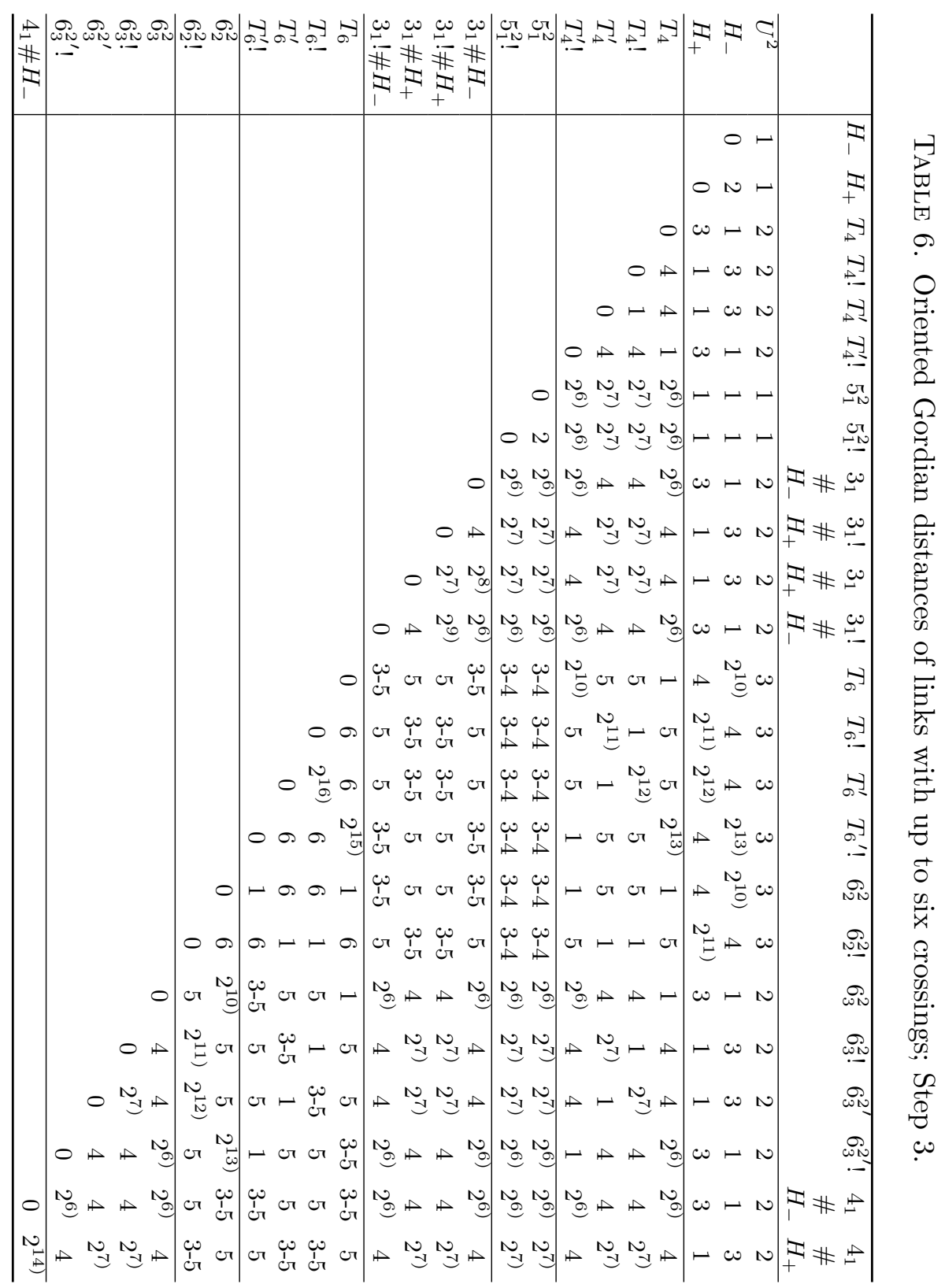


[8] Yasuyuki Miyazawa, Gordian distance and polynomial invariants, J. Knot Theory Ramifications 20 (2011), 895-907.

[9] Yasuyuki Miyazawa, The Jones polynomial of an unknotting number one knot, Topology Appl. 83 (1998), no. 3, 161-167.

[10] Kunio Murasugi, On a certain numerical invariant of link types, Trans. Amer. Math. Soc. 117 (1965), 387-422.

[11] Kunio Murasugi, On the signature of links, Topology 9 (1970), 283-298.

[12] Dale Rolfsen, Knots and links, AMS Chelsea Press, Providence, RI, 2003, Originally published: Berkeley, CA: Publish or Perish Inc. 1976.

[13] Alexander Stoimenow, Polynomial values, the linking form and unknotting numbers, Math. Res. Lett. 11 (2004), no. 5-6, 755-769.

[14] Toshifumi Tanaka, Signed Gordian distances, the Jones polynomial and Rasmussen invariant of knots, Sci. Rep. Fac. Educ. Gifu Univ. (Nat. Sci.) 38 (2014), 5-14.

[15] Ichiro Torisu, The determination of the pairs of two-bridge knots or links with Gordian distance one, Proc. Amer. Math. Soc. 126 (1998), no. 5, 1565-1571.

[16] Paweł Traczyk, A criterion for signed unknotting number, Low-dimensional topology (Funchal, 1998), Contemp. Math., vol. 233, Amer. Math. Soc., Providence, RI, 1999, pp. 215-220.

Department of Mathematics, Kobe University, Rokko, Nada-ku, Kobe 657-8501, Japan

E-mail address: s.kanenob@gmail.com

Department of Mathematics, Osaka City University, Sugimoto, Sumiyoshi-ku, Osaka 5588585, JAPAN

E-mail address: kanenobu@sci.osaka-cu.ac.jp 